
allemande

\title{
Max Weber et la question de la démocratisation des institutions politiques de l'Allemagne (1917-1918)
}

Christian Baechler

\section{(2) OpenEdition}

1 Journals

Édition électronique

URL : https://journals.openedition.org/allemagne/573

DOI : 10.4000/allemagne.573

ISSN : 2605-7913

Éditeur

Société d'études allemandes

\section{Édition imprimée}

Date de publication : 29 décembre 2017

Pagination : 315-332

ISSN : 0035-0974

\section{Référence électronique}

Christian Baechler, « Max Weber et la question de la démocratisation des institutions politiques de I'Allemagne (1917-1918) », Revue d'Allemagne et des pays de langue allemande [En ligne], 49-2 | 2017, mis en ligne le 29 décembre 2018, consulté le 18 mai 2021. URL : http://journals.openedition.org/ allemagne/573 ; DOI : https://doi.org/10.4000/allemagne.573 


\section{Max Weber et la question de la démocratisation des institutions politiques de l'Allemagne (1917-1918)}

\section{- Christian Baechler*}

Avec la prolongation de la guerre, la «paix civile» (Burgfrieden) d'août 1914 se fissure progressivement. Les partis politiques et l'opinion allemande se divisent sur les buts de guerre et sur la question de la guerre sous-marine sans restriction. Le débat, mal contenu par la censure, déborde sur la place publique après son assouplissement en novembre 1916. Les partis exigent une participation plus active du Reichstag à la définition de la politique du chancelier Bethmann-Hollweg, jugée trop faible par certains et trop indécise pour la plupart. À la suite d'un hiver 1916/1917 particulièrement rigoureux, de la révolution de février (8-13 mars) en Russie et des premiers troubles sociaux importants à l'annonce d'une nouvelle réduction de la ration de pain, la question de la réforme du mode de scrutin en Prusse fait l'objet de discussions de plus en plus vives. Le débat sur les buts de guerre et sur la réforme en Prusse aboutit finalement à la chute de Bethmann-Hollweg en juillet 1917, sous la pression conjointe du haut commandement militaire et du Reichstag. C'est le début d'une période plus agitée à l'intérieur et d'une remise en cause du régime constitutionnel ${ }^{(1)}$. Animé d'un ardent patriotisme, Max Weber, qui s'est prononcé contre les annexions en Europe et la guerre sous-marine sans restriction, intervient avec passion dans le débat sur la réforme des institutions à partir de mars 1917 avec pour objectif principal le renforcement du front intérieur.

\section{La question de la réforme du mode de scrutin en Prusse}

La durée de la guerre et les énormes sacrifices consentis par la population posent de manière aiguë la question de la réforme du système électoral prussien inégal. La chambre basse du Landtag est en effet élue au suffrage universel, indirect, inégal et

* Professeur émérite en histoire contemporaine, Université de Strasbourg.

1 Pour le contexte général, cf. Christian BAechler, L'Allemagne et les Allemands en guerre 1914-1918, Paris, 2016, p. 357-408. 
public. Les électeurs sont répartis en trois classes, en fonction de leur imposition, les plus imposés étant placés dans la première classe, chaque classe correspondant à un tiers du montant global des impôts. Lors des élections de 1913, la première classe comptait ainsi 4,43\% des électeurs, la deuxième $15,76 \%$ et la troisième $79,81 \%$. Selon une procédure publique, chaque classe élit le même nombre de grands électeurs, les grands électeurs élisant les députés. Comme, de plus, le découpage des circonscriptions avantage l'Est prussien, les conservateurs ont toujours au moins $45 \%$ des mandats depuis $1885^{(2)}$. Avec le soutien des groupes parlementaires du Zentrum et du parti national libéral, bien plus conservateurs qu'au Reichstag, la Chambre basse est dominée par une majorité conservatrice. Comme la Chambre haute (Herrenhaus) est composée de membres de droit et de membres nommés par le roi, le Landtag prussien est un bastion du conservatisme. Grâce à son droit de veto au conseil fédéral (Bundesrat), la Prusse peut bloquer toute évolution du Reich vers un régime parlementaire.

Lors du discours du trône du 13 janvier 1916, Guillaume II fait allusion à un changement «des principes pour la représentation du peuple dans les corps législatifs» pour l'après-guerre. C'est la vague formule de compromis adoptée par un ministère prussien très divisé sur l'opportunité d'annoncer une réforme ${ }^{(3)}$. Mais, début 1917, sous la pression de l'opposition interne, le SPD menace de ne pas voter les crédits de guerre s'il n'y a pas de réforme immédiate. Contraint d'agir pour préserver le Burgfrieden, Bethmann-Hollweg annonce au Reichstag, le 27 février, puis au Landtag prussien, le 14 mars, une «nouvelle orientation» traduisant dans de nouvelles formes institutionnelles l'unité morale de la nation dans la guerre. Le 5 avril, il déclare au ministère prussien que la situation politique intérieure et extérieure exige «un acte clair pour l'opinion publique avec la promesse d'un suffrage universel, égal, secret et direct pour la Prusse. [...] La monarchie doit se présenter comme une monarchie populaire» ${ }^{(4)}$. Dans son «message de Pâques» du 7 avril 1917, Guillaume II annonce une réforme du suffrage en Prusse "pour créer les bases d'une collaboration libre et joyeuse de tous les membres de notre peuple». Il demande au chancelier de préparer immédiatement une réforme qui sera appliquée lorsque les soldats rentreront dans leurs foyers. Il ajoute qu'il n'y a plus place pour un suffrage par classes et se prononce pour un scrutin direct et secret. Il n'est pas question de suffrage égal ${ }^{(5)}$. Mais, sous la pression de grèves dans la métallurgie, le SPD adopte les 18 et 19 avril une résolution exigeant «l'élimination immédiate des inégalités de droits civiques dans le Reich, les États et les communes » ${ }^{(6)}$. Il se déclare aussi en accord avec la résolution du congrès des soviets d'ouvriers et de

2 G.A. Ritter, Wahlgeschichtliches Arbeitsbuch. Materialien zur Statistik des Kaiserreichs 1871-1918, Munich, 1980, p. 132-149.

3 Die Protokolle des Preußischen Staatsministeriums 1817-1934/38, vol. 10 (1909-1918), Hildesheim et al., 1999, documents n ${ }^{\circ} 153$ et 154.

4 Protokolle des Preußischen Staatsministeriums, ${ }^{\circ} 198$.

5 Lors du conseil des ministres de Prusse du 6 avril, Bethmann-Hollweg précise que l'empereur n'annoncera pas le suffrage égal, afin d'éviter de dures controverses pendant la guerre, Protokolle des PreuBischen Staatsministeriums, $\mathrm{n}^{\circ}$ 199. Les groupes parlementaires des conservateurs, des conservateurs libres, du Zentrum et des nationaux libéraux sont pour un vote plural, afin de limiter l'influence du SPD, ibid., nº 202, séance du 23 mai 1917.

6 Erich Matthias, Eberhard Pikart, Die Reichstagsfraktion der deutschen Sozialdemokratie 1898 bis 1918, Düsseldorf, 1966, t. II, $\mathrm{n}^{\circ} 439$. 
soldats russe pour une paix sans annexion et sans indemnité, établissant implicitement une relation entre les réformes intérieures et la paix.

C'est dans ce contexte que Max Weber intervient dans le débat public. Dès le 19 mars 1917, il écrit à la rédaction de la Frankfurter Zeitung qu'il faut mettre à l'ordre du jour la lutte contre le suffrage prussien: "Après le déroulement des événements en Russie, il faut compter avec certitude sur un durcissement de ton chez les sociaux-démocrates » ${ }^{(7)}$. Il publie le 28 mars dans le quotidien libéral de gauche un article intitulé: "Une loi électorale d'urgence du Reich. Le droit des combattants de retour à la maison » ${ }^{(8)}$. Il invite les partis «vraiment nationaux» à déposer immédiatement une proposition de loi au Reichstag, accordant à tous les hommes incorporés dans l'armée, à leur majorité, le droit de vote dans la classe supérieure des assemblées élues des États, si le suffrage universel n'est pas égal ${ }^{(9)}$. Il souligne qu'avec le système prussien des trois classes les combattants, quelle que soit leur condition sociale, perdraient du poids électoral du fait de leur appauvrissement relatif par rapport à ceux qui n’ont pas été incorporés dans l'armée. Ce serait un «scandale», car la "ploutocratie» serait renforcée: "Les élections de la paix mettraient en place en Prusse un parlement de parvenus de guerre, qui déciderait de notre avenir, et alors ce serait trop tard.» Il conclut que "l'armée qui a combattu doit aussi avoir une voix décisive dans la reconstruction de la patrie après la guerre».

Le 26 avril, Max Weber publie un nouvel article dans la Frankfurter Zeitung sur «le Landtag prussien et le Reich", pour répondre aux critiques de journaux berlinois qui affirment que le suffrage prussien ne concerne pas le Reich. Par une analyse détaillée de la constitution de 1871 et de son fonctionnement, il montre que, du fait de la position hégémonique de la Prusse, le Landtag prussien joue un rôle décisif pour tous les actes importants de la fédération. Le gouvernement du Reich est ainsi dans la position inconfortable de devoir s'adapter au contrôle du Reichstag et du Landtag prussien, dont la composition et les intérêts sont très différents, d'où un double langage qui entache la réputation de la Couronne. Dans cet édifice fédéral complexe, le Landtag prussien a une position privilégiée, alors que les Landtage des autres États sont en quelque sorte «médiatisés». Le Reich est de fait sous l'influence d'une "petite clique de privilégiés du suffrage prussien». Le suffrage prussien concerne ainsi l'ensemble du Reich. La voix prépondérante de la Prusse au Bundesrat doit être instruite par le peuple prussien et non par une caste de privilégiés qui s’appuie sur un «suffrage ploutocratique».

Max Weber publie dans la revue Europäische Staats- und Wirtschafts-Zeitung du 21 avril un long article sur «le droit de vote prussien», rédigé avant le "message de Pâques » de l'empereur, où il développe ses arguments sur un ton souvent très violent,

7 Max Weber Gesamtausgabe, Abteilung II, Briefe, vol. 9, Gerd Krumeich, M. Rainer Lepsius (éd.), Max Weber Briefe 1915-1917, Tübingen, 2008, 19.03.1917.

8 «Ein Wahlrechtsnotgesetz des Reiches. Das Recht der heimkehrenden Krieger », Max Weber Gesamtausgabe, Abteilung I, Schriften und Reden, vol. 15, Wolfgang J. Mommsen, Gangolf Hübinger (éd.), Max Weber. Zur Politik im Weltkrieg. Schriften und Reden 1914-1918, Tübingen, 1984, p. $217-221$. Dorénavant cité sous MWG.

9 Max Weber explique dans une lettre du 3 février au député progressiste Friedrich Naumann qu'une telle loi «résout en pratique la question du suffrage et intervient formellement, seulement temporairement dans la constitution», ibid., p. 216. 
qui témoigne de son tempérament volcanique ${ }^{(10)}$. Soulignant qu'on ne peut prévoir la fin de la guerre du fait de l'intervention des États-Unis, il estime que «la réforme immédiate et sans délai du suffrage prussien est une nécessité pressante». Il faut régler le problème dès à présent, car on ne pourra imposer la réforme aux privilégiés après guerre, à moins de l'octroyer. Il attaque violemment la majorité "ploutocratique» du Landtag prussien qui a témoigné de son égoïsme depuis le début de la guerre ${ }^{(11)}$ et de sa démagogie dans les buts de guerre: «Toute autre solution en Prusse que le droit de suffrage du Reichstag serait considérée objectivement par les combattants de retour dans leurs foyers - et c'est la question - comme une duperie.» Il écarte toutes les formes de vote plural envisagées. Il est particulièrement violent à l'encontre des projets favorisant les diplômés des «milieux d'écrivassiers [Litteraten] »: «Il n’y a pas en Allemagne de milieu en moyenne aussi peu qualifié politiquement. [...] Ce milieu est le dernier auquel on doive accorder des droits particuliers dans le vote populaire. » Les membres de la Bildung sont qualifiés pour l'érudition, la fonction publique ou la technique, mais pas pour être des hommes politiques responsables. Ces privilégiés avec leurs «sinécures» et leurs "prébendes» sont moins qualifiés pour la politique que les employés et les ouvriers de sociétés privées soumises à la concurrence du marché, qui ont une expérience concrète des luttes politiques. Il écarte aussi le vote plural en fonction de la situation familiale, de la propriété - c'est avantager «le nombre gigantesque des nouveaux rentiers de la guerre» - et de la profession. Le vote plural continuerait à empoisonner la vie politique: «Seul le droit de suffrage du Reichstag mettra fin aux luttes sur le droit de suffrage. La fin de ces luttes est la condition d'un travail concret. »

Max Weber précise, reprenant des thèses déjà développées avant-guerre, que le but de la démocratisation est l'unité nationale et la sélection d'hommes politiques responsables pour une Allemagne plus forte à l'extérieur: «La “démocratie” n’a jamais été un but en soi pour le signataire. Ce qui l'a seulement intéressé et l'intéresse seulement, c'est de rendre possible une politique nationale réaliste pour une Allemagne unie et forte à l'extérieur.» Si l'on ne réforme pas, ce sera la continuation de décennies d'une politique bureaucratique prussienne sans succès. Le but final de la réforme est de permettre la sélection de dirigeants politiques responsables: «On a besoin aujourd'hui de la libre scène du vote populaire universel pour fait naître ces dons politiques spécifiques qui non seulement ne sont pas identifiables par des diplômes ou autre méthode censitaire, mais que ces privilèges empêchent de croître. Le vote populaire libre et égal et la sélection au sein du parlement sont le seul contrepoids possible à la domination sans limite du pur spécialiste. Le pouvoir inévitable et supérieur du spécialiste, personnifié par les fonctionnaires publics [Beamtenschaft], s'affirme suffisamment et souvent plus que suffisamment face au parlement, quelle qu'en soit la nature.» Il s'agit de trouver un lieu, le parlement, où le spécialiste de la fonction publique a le moins d'influence possible sur la sélection des leaders politiques, «car ce ne sont pas des qualités et des connaissances techniques qui qualifient l'homme politique pour ses

10 «Das preußische Wahlrecht», MWG, I, 15, p. 224-235. La revue a été fondée en mars 1916 par Heinrich von Frauendorfer et Edgar Jaffé. Jaffé est co-éditeur avec Weber de l'Archiv für Sozialwissenschaft und Sozialpolitik.

11 Max Weber a très violemment critiqué le projet de loi prussien sur l'extension des fidéicommis de janvier 1917 dans la Frankfurter Zeitung du $1^{\text {er }}$ mars 1917. 
missions». On retrouve ici un thème majeur du sociologue: la liberté menacée par l'emprise quasi inévitable de la bureaucratie dans la société moderne, la sélection de leaders politiques responsables par le biais des luttes entre partis pour lui faire contrepoids ${ }^{(12)}$. Max Weber estime aussi que ces leaders politiques sont seuls capables de mobiliser les masses à l'heure de la démocratie et de mener ainsi une politique nationale de grande puissance.

Max Weber revient sur la question du suffrage en Prusse en décembre 1917 dans une brochure publiée dans une collection lancée par la revue Die Hilfe de Friedrich Naumann ${ }^{(13)}$. La brochure a probablement été rédigée en septembre pour répondre au projet de suffrage professionnel des conservateurs prussiens. Max Weber reprend les arguments développés dans son article du 21 avril contre le vote plural, en ajoutant des réflexions d'ordre économique et sociologique. Il souligne qu'il n'est pas possible de délimiter avec précision les professions dans une société moderne complexe. Il rejette l'idée de favoriser les classes moyennes indépendantes, le Mittelstand, car ce serait faire obstacle à l'intensification et à la rationalisation de l'économie qui sera nécessaire pour payer les dettes de guerre. Il rejette aussi les idées à la mode de «Solidaritätswirtschaft» ou de "Gemeinschaftswirtschaft», nées de l'expérience de guerre. L'économie de guerre a fait naître un "capitalisme de brigandage» avec des bénéfices énormes et sans risques, sans l'éthique du capitalisme, une économie où le capitalisme privé ne fait plus contrepoids à la bureaucratie étatique. Il souligne aussi que le droit de vote égal est un contrepoids aux inégalités de fortune et de formation (Bildung) des citoyens, ainsi que, par le biais d'un parlement exprimant les besoins des masses, un contrepoids à une économie qui sera plus fortement cartellisée après-guerre.

Il termine en rejetant l'objection selon laquelle le parlementarisme serait une corruption de la démocratie et la domination d'une clique. La démocratie directe n'étant pas possible dans un grand pays, le système parlementaire est le seul moyen pour contrôler la bureaucratie: «Il n'y a qu'une alternative: un "État autoritaire [Obrigkeitsstaat]" bureaucratique avec un parlementarisme de façade où la masse des citoyens est sans droits, sans liberté et "administrée" comme un troupeau de vaches, ou incorporer les citoyens dans l'État comme co-souverains. Un peuple de maîtres [Herrenvolk] n'a de ce point de vue pas le choix - et seul un peuple de maîtres peut et doit mener une politique mondiale.» Ne pas appliquer le suffrage universel égal, c'est compromettre l'avenir de la nation, orienter les forces populaires contre l'État et renoncer à mobiliser la nation dans une nouvelle guerre défensive. On retrouve un thème déjà ancien chez Max Weber, la nécessité d'intégrer l'ensemble de la nation par des réformes pour mener une politique mondiale ${ }^{(14)}$. Les grèves de janvier 1918 confirment cette nécessité. Dans trois articles du début février, il déclare que les attaques contre le projet de

12 En particulier dans Wirtschaft und Gesellschaft, $2^{\mathrm{e}}$ partie, chap. IX «Sociologie du pouvoir». Pour replacer les écrits de guerre de Max Weber dans son œuvre sociologique, nous renvoyons au remarquable article de François CHAzEL, «Les Écrits politiques de Max Weber: un éclairage sociologique sur des problèmes contemporains ", Revue française de sociologie, 46/4 (2005), p. 841-870.

13 Wahlrecht und Demokratie in Deutschland, MWG, I, 15, p. 344-396. Un bref résumé paraît dans Die Hilfe dès le 29 novembre.

14 Cf. Wolfgang J. Mommsen, Max Weber et la politique allemande 1890-1920, Paris, 1985 (texte allemand de 1974), p. 59-97. 
loi sur la réforme du suffrage en Prusse sont la cause principale des grèves ${ }^{(15)}$. Refuser la réforme, c'est empêcher la victoire de l'Allemagne et compromettre son avenir: «Est national l'homme politique qui considère la politique intérieure du point de vue de son adaptation inévitable aux devoirs de politique extérieure. Celui qui n'accepte pas les conséquences "démocratiques" qui en découlent renonce à la politique de grande puissance qui les rend inévitables» ${ }^{(16)}$.

\section{La question de la parlementarisation du Reich}

On constate depuis le début de 1916 une pression croissante des partis politiques pour un renforcement du rôle du Reichstag, en particulier dans la définition des buts de guerre. Une ordonnance impériale du 4 novembre 1916 autorise la commission budgétaire du Reichstag à se réunir entre les sessions pour discuter des questions politiques intérieures et extérieures. Elle devient la commission principale (Hauptausschuß) du Reichstag, un lieu de discussion et de concertation entre les groupes parlementaires. Le 30 mars 1917, le Reichstag va plus loin et décide de créer une commission de réflexion sur les questions constitutionnelles. Réunie pour la première fois le 2 mai, la commission propose plusieurs réformes: le contreseing du ministre de la Guerre pour les nominations d'officiers par l'empereur, la possibilité pour un parlementaire d'être chancelier ou secrétaire d'État sans renoncer à son mandat, une procédure d'accusation du chancelier, sans aller jusqu'à une réelle responsabilité du ministère devant le Reichstag, le redécoupage des circonscriptions électorales, la réforme du suffrage dans les États fédérés. En dehors du SPD, de l'aile gauche du Zentrum, d'une fraction de progressistes et de quelques nationaux libéraux, on ne souhaite pas remettre en cause les principes du système constitutionnel, mais instaurer un meilleur équilibre entre le Reichstag et l'exécutif. Face à l'opposition du chancelier, de Guillaume II $^{(17)}$, du ministère prussien ${ }^{(18)}$ et des États fédérés, qui craignent un affaiblissement du Bundesrat et une remise en cause du système fédéral ${ }^{(19)}$, la commission renonce à ses propositions.

À la veille de la réunion de la commission constitutionnelle dont il est membre, le député progressiste Conrad Haußmann demande à Max Weber quelles sont les réformes à proposer. Dans une lettre du 29 avril, ce dernier mentionne trois points qu'il estime importants: le renforcement du pouvoir du Reichstag et du

15 MWG, I, 15, p. 404-420. Articles de la Frankfurter Zeitung des 3, 5 et 7 février 1918.

16 Ibid., p. 406, article du 3 février. Les grèves sont aussi la conséquence de la campagne d'annexions à l'Est, financée par les puissances de l'argent, qui nourrit une démagogie de boulevard, «la plus sauvage des démagogies sans démocratie, bien plus, à cause du manque de démocratie», ibid., p. 413, article du 7 février.

17 Kurt Riezler note le 9 mai que la proposition sur la nomination des officiers, qui touche au pouvoir de commandement de l'empereur, ébranle la position du chancelier auprès de celui-ci, cf. Karl Dietrich Erdmann (éd.), Kurt Riezler. Tagebücher, Aufsätze, Dokumente, Göttingen, 1972.

18 Protokolle des Preußischen Staatsministeriums, $n^{\circ} 201$, séance du $1^{\mathrm{er}}$ mai 1917.

19 La Commission des Affaires étrangères du Bundesrat, réunie le 9 mai, s'inquiète de l'immixtion du Reichstag dans les questions constitutionnelles. Elle se prononce contre toute réforme durant la guerre et pour le respect du fédéralisme, cf. Ernst Deuerlein, Das Bundesratsausschuß für die auswärtigen Angelegenheiten 1870-1918, Ratisbonne, 1955, p. 295-297. 
chancelier ${ }^{(20)}$; l'institution d'un droit d'enquête du Reichstag avec obligation de témoigner pour les fonctionnaires publics; le contrôle des déclarations du monarque, car «l'exclusion de ce facteur de notre politique est vraiment une question vitale» ${ }^{(21)}$. Max Weber a fait des propositions sur ces deux derniers points dès 1908, après l'affaire du Daily Telegraph ${ }^{(22)}$. Début mai, il fait parvenir à Haußmann des textes de projet de loi $^{(23)}$. Dans l'argumentaire accompagnant le projet sur le droit d'enquête du Reichstag, il souligne que la croissance du pouvoir des bureaucraties publiques est un phénomène général. Les fonctionnaires agissent sans contrôle et sans responsabilité, car non seulement ils ont la compétence technique (Fachwissen), la connaissance des dossiers et des procédures (Dienstwissen), mais peuvent aussi se retrancher derrière le «secret administratif» pour ne pas les partager. Le secret administratif étant la base de leur pouvoir, il faut assurer la publicité des questions n'exigeant pas le secret. Lorsque le secret est indispensable, il doit être partagé avec des instances parlementaires de contrôle, une commission où sont représentés les partis politiques. Le droit d'enquête est, pour lui, le pouvoir le plus efficace du parlement face à la bureaucratie, tandis que la publicité permet d'éduquer les citoyens aux affaires administratives courantes.

Max Weber envoie aussi un projet sur la suppression de l'article 9, paragraphe 2 de la constitution, qui stipule que "personne ne peut être en même temps membre du Bundesrat et du Reichstag». C'est l'obstacle qui ne permet pas à un chancelier ou à un secrétaire d'État d'être membre du Reichstag ${ }^{(24)}$. Il se prononce aussi pour la création d'un Conseil de la Couronne du Reich (Reichskronrat), présidé par l'empereur et composé de représentants des États allemands et du Reichstag, pour conseiller sur les questions de haute politique. Il s'agit d'empêcher les déclarations intempestives de Guillaume II: "Cette situation doit être absolument écartée, sinon nous irons à nouveau vers le même sort après-guerre, d'autant qu'il est notoire que le successeur au trône [le Kronprinz Wilhelm] montre des tendances tout à fait semblables et donne l'impression d'une totale incapacité à apprendre " ${ }^{(25)}$. Il propose enfin un projet de loi permettant de condamner «la publication [non autorisée] de discours et télégrammes monarchiques".

À la suite de la suspension des séances de la commission constitutionnelle où les progressistes ont proposé sans succès la suppression du paragraphe 9,2, Max Weber publie, en mai et en juin, une série de quatre articles sur la réforme constitutionnelle du Reich dans la Frankfurter Zeitung. Remaniés, élargis et complétés, ces articles seront publiés sous forme de brochure en mai 1918, sous le titre Parlement et gouvernement

20 Weber parle d'accroissement «formel» du pouvoir de l'empereur face au Bundesrat, afin de renforcer le Reichstag et le chancelier, celui-ci étant seul responsable de l'instruction de la "voix présidentielle » prussienne au Bundesrat.

21 Lettre du 29 avril 1917, MWG, II, 9.

22 W.J. Mommsen, Max Weber et la politique allemande 1890-1920 (note 14), p. 200-201.

23 Lettres des $1^{\text {er }}$ et 5 mai 1917, MWG, II, 9; MWG, I, 15, p. 268-277, pour les projets.

24 L'article 15 de la constitution précise en effet que le chancelier préside le Bundesrat. Selon la pratique, les principaux secrétaires d'État du Reich sont aussi ministres prussiens et plénipotentiaires au Bundesrat. 
dans l'Allemagne réorganisée. Contribution à une critique politique de la bureaucratie publique et des partis ${ }^{(26)}$. Dans l'article du 27 mai sur «l'héritage de Bismarck», Max Weber se livre à une violente critique de sa politique intérieure qui a divisé pour régner, détruit le parti national libéral, représentant du parlementarisme et d'une politique unitaire, et empêché le développement du SPD et des syndicats: «Il a laissé une nation sans aucune éducation politique, nettement en dessous du niveau qu'elle avait atteint à cet égard vingt ans plus tôt. Et surtout, une nation sans aucune volonté politique, habituée à ce que le grand homme d'État à sa tête se préoccupe pour elle de la politique » ${ }^{(27)}$. Le résultat est un parlement impuissant, d'un niveau intellectuel très bas, non du fait de la médiocrité des hommes, mais parce qu'il n'a pas de pouvoir de décision et, de ce fait, ne forme pas de véritables hommes politiques.

Dans les articles des 9 et 10 juin, il aborde à nouveau un thème essentiel de son œuvre scientifique: le phénomène général de rationalisation et de bureaucratisation de la société moderne ${ }^{(28)}$. De même que les progrès du capitalisme sont la mesure de la modernisation de l'économie, les progrès de la bureaucratie sont la mesure de la modernisation de l'État de masse, qu'il soit monarchique ou démocratique. La bureaucratie étend partout son pouvoir, dans les grandes entreprises, dans l'armée et même dans les partis politiques « avec la rationalisation croissante de leur technique de campagne électorale» et l'apparition de permanents. C'est un processus universel auquel on ne peut échapper. Il n'y a pas, de plus, d'exemple historique de disparition de la bureaucratie là où elle règne en maître, sauf en cas d'effondrement de la civilisation. La bureaucratie moderne est encore plus puissante, car spécialisée et formée pour régler les tâches de la vie quotidienne: "Aujourd'hui une "socialisation" croissante signifie inévitablement en même temps une bureaucratie croissante» ${ }^{(29)}$. Aussi, «dans un État moderne, le vrai pouvoir [Herrschaft], qui s'exerce [...] dans l'application de l'administration au quotidien, est nécessairement et inévitablement aux mains des fonctionnaires» ${ }^{(30)}$. La bureaucratie étatique règnerait sans partage s'il n'y avait pas le contrepoids du capitalisme privé.

Face à la marche inéluctable de la bureaucratie, l'organisation politique doit répondre à trois questions: comment préserver un reste de liberté individuelle, comment contrôler l'administration étatique et produire des hommes politiques responsables? Max Weber constate que le monarque n'est pas un contrepoids à la bureaucratie, car il n'a ni la capacité technique pour la contrôler, ni la formation d'un homme politique, l'expérience des luttes partisanes lui étant interdite. Même s'il pense gouverner, c'est la bureaucratie qui dirige sans contrôle et sans responsabilité. Depuis la retraite de Bismarck, l’Allemagne est ainsi dirigée par des fonctionnaires intègres, bien formés,

26 Parlament und Regierung im neugeordneten Deutschland. Zur politischen Kritik des Beamtentums und Parteiwesens, Munich, Duncker \& Humblot, 1918. Le manuscrit, achevé fin septembre, inclut l'article du 26 avril 1917 sur la question du suffrage en Prusse. La publication est retardée par la censure et par des problèmes matériels, cf. MWG, I, 15, p. 432-596. Nous tirons les citations du texte définitif publié en mai 1918. Sous le titre général de «Beamtenschaft und politische Führertum». 
intelligents, disciplinés, mais pas par des hommes politiques responsables, d'où une politique extérieure désastreuse. Le seul contrepoids possible à la bureaucratie étatique est un parlement qui n'a pas seulement le pouvoir de refuser le budget et les projets de loi, mais le pouvoir de faire sortir de son sein le chef de l'administration ou du moins de provoquer son départ et de contraindre à mener une politique correspondant aux vœux de la majorité parlementaire. Les chefs de partis sont alors des collaborateurs positifs de la puissance publique. L'exemple des chefs de grandes entreprises montre qu'il ne manque pas de natures de chef (Führer) en Allemagne, ce qui manque, c'est la lutte politique, car «l'essence de toute politique, c'est la lutte, le recrutement d'alliés et de partisans qui suivent de leur plein gré» tant que le leader a du succès: "Un parlement de travail, et non pas de discours, constitue seul le terrain sur lequel poussent non seulement des qualités de chef démagogique, mais de chef vraiment politique et progressant par le biais de la sélection. Mais un parlement qui agit est un parlement qui contrôle en permanence en collaborant avec l'administration. Ce n'était pas le cas chez nous avant la guerre. Après la guerre, le parlement doit être transformé dans ce sens, sinon ce sera à nouveau la même misère » ${ }^{(31)}$. Le parlement est ainsi instrument de contrôle de l'administration et lieu de sélection des dirigeants politiques.

Dans le dernier article publié le 24 juin, Max Weber reprend les propositions qu'il développait dans sa correspondance avec Conrad Haußmann ${ }^{(32)}$. Pour contrôler l'administration, il faut doter le parlement d'un droit d'enquête par des commissions spécialisées, qui permettent aussi de former des hommes politiques compétents. Il revient sur la nécessité de supprimer le paragraphe 9,2, afin que les chefs de parti prennent la responsabilité de la politique du Reich et que leur parti en assume la responsabilité au Reichstag. Il rejette l'argument selon lequel le parlementarisme irait à l'encontre du fédéralisme, soulignant qu'il renforcerait au contraire les États allemands face à la puissance hégémonique de la Prusse. Il permettrait aussi de subordonner l'autorité militaire à l'autorité politique pour tout ce qui n'est pas strictement militaire et d'écarter ainsi une influence qui a été néfaste pour l'Allemagne. Il consacre un long développement à une virulente critique de la politique extérieure allemande depuis le départ de Bismarck, en particulier aux déclarations théâtrales de Guillaume II. La direction politique du Reich a permis et même contribué aux déclarations de l'empereur, et les a utilisées comme moyens de la diplomatie, alternant les gestes énergiques et les reculs, ce qui est pour beaucoup dans l'attitude anglaise et l'isolement allemand de 1914. Ces déclarations ont aussi accrédité à l'étranger la thèse qu'il faut libérer l'Allemagne de l'autocratie. Le monarque étant incontrôlable, une loi doit sanctionner le responsable politique, c'est-à-dire le chancelier. Mais pour qu'il assume la responsabilité, il faut une concertation préalable sur la publication des déclarations de l'empereur dans le cadre d'un conseil de la Couronne du Reich.

Max Weber termine par une critique acerbe de ceux qui prétendent que le peuple allemand n'est pas mûr politiquement, en particulier les «écrivassiers» (Litteraten): «Les écrivassiers allemands d'aujourd'hui sont les derniers à pouvoir porter un jugement sur la "maturité" politique. Ils ont collaboré par leurs acclamations à presque toutes

31 Ibid., p. 486.

32 «Verwaltungsöffentlichkeit und politische Verantwortung». 
les erreurs de la politique allemande d'avant-guerre ${ }^{(33)}$. Si les Allemands manquent d'assurance à l'extérieur, c'est qu'ils ont l'habitude d'être dominés à l'intérieur: «La “volonté d’impuissance" à l'intérieur, prêchée par les écrivassiers, n’est pas compatible avec la "volonté de puissance" dans le monde, proclamée avec tant de bruit» ${ }^{(34)}$. Et il déclare que «seul un peuple politiquement mûr est "un peuple de maîtres": un peuple qui garde dans ses mains le contrôle de l'administration de ses affaires et qui détermine de manière décisive par ses représentants élus le choix de ses dirigeants politiques » ${ }^{(35)}$.

Cette série d'articles a un large écho, en particulier les violentes attaques contre le régime personnel de Guillaume II. Le numéro du 24 juin est saisi, mais trop tard, tandis que la Frankfurter Zeitung est soumise à une censure préalable jusqu'au 4 juillet, censure suspendue à la suite d'une question au gouvernement du député Haußmann. L'historien Friedrich Meinecke est sollicité par le chancelier von Bethmann-Hollweg pour répondre aux articles qui ont eu un effet désastreux sur Guillaume $\mathrm{II}^{(36)}$. Kurt Riezler, conseiller et confident du chancelier, déclare ainsi le 25 à Haußmann que l'ambiance favorable à la nouvelle orientation politique en a été refroidie: "Malheureusement, la Frankfurter Zeitung a brisé, avec un article tout à fait indéfendable de Max Weber, une marmite préparée avec beaucoup de peine; nous l'entourons et gémissons sur la soupe répandue; les adversaires se réjouissent sans retenue.» Dans une lettre du 28 juin à Hans Delbrück, l'influent directeur des Preußische Jahrbücher, Max Weber justifie son article en insistant sur la nécessité absolue d'écarter l'influence du monarque, en tout cas ses déclarations publiques: «Le monarque est incorrigible et ce qu'on peut attendre du fils chacun le sait, et le chancelier mieux que tous. Il faut en tirer les conséquences, que l'on pense ce que l'on veut du "parlementarisme". Si la nation, sous l'influence de ses publicistes, maintient sa "volonté d'impuissance" à l'intérieur, alors il ne faudra plus parler de politique extérieure, car peu importera alors ce que l'on entreprendra: tout sera gâté comme depuis 25 ans. Ce que ce système a de plus capable - les militaires - manque politiquement autant de jugement que tous les autres [...]. Le parlementarisme le plus corrompu ne peut - d'un point de vue purement politique! - fonctionner plus mal. Je ne vois pas d'autre voie devant moi que de dire dorénavant sans précaution “ce qui est". C'est: la destruction de notre avenir politique par la dynastie et ses panégyristes, intéressés ou désintéressés " ${ }^{(37)}$. Revenant sur l'article et sur la chute de Bethmann-Hollweg, Conrad Haußmann écrit à Max Weber, le 3 septembre: «En tout cas, votre article a créé l'atmosphère qui a mené en juillet à une forte, mais encore insuffisante décharge [Entladung] » ${ }^{(38)}$.

Dans la brochure publiée en mai 1918, dont le manuscrit est prêt dès septembre 1917, Max Weber répond aux objections à l'encontre des réformes proposées dans ses articles. Dans la préface ${ }^{(39)}$, il attaque violemment les universitaires et les écrivassiers

Ibid., p. 592.

Ibid., p. 595.

Ibid., p. 525.

Friedrich Meinecke, Straßburg, Freiburg, Berlin 1901-1919. Erinnerungen, Stuttgart, 1949, p. $224-225$.

Ibid., lettre du 28 juin 1917.

MWG, II, 9, p. 763, lettre de Max Weber à Georg Hohmann du 3 septembre 1917, note 4.

MWG, I, 15, p. 433-436. 
qui s'efforcent, depuis 40 ans, de discréditer la représentation populaire. Il rejette la thèse qu'ils défendent depuis le début de la guerre d'une opposition entre une conception spécifiquement allemande de l'État et celle des pays occidentaux. Dans l'État moderne de masse, il n'y a qu'un nombre restreint de solutions techniques: aucune ne remet en cause la germanité. Le parlementarisme n'est pas étranger à la tradition allemande comme ils le prétendent, mais prendra d'autres formes en Allemagne. Dans un chapitre ajouté sur les relations entre "parlementarisation et démocratisation " ${ }^{(40)}$, il souligne que le pouvoir du parlement n'est pas menacé en soi par le suffrage universel, comme le craignent certains, même s'il donne de l'importance à l'appareil des partis à cause de la nécessité d'une propagande de masse. La "démocratisation active» des masses a des conséquences sur la sélection des «chefs » (Führer). La reconnaissance des notables n'est plus déterminante dans l'ascension du «chef» politique, mais l'acclamation des masses, «la confiance et la foi que les masses placent en lui»: «Dans l'essence des choses, cela signifie un tournant césariste dans la sélection des chefs. Et le fait est que toute démocratie penche dans ce sens. » Mais il ajoute que l'on constate la même évolution césariste dans les monarchies, même si cette évolution y est modérée. Il y a un principe césariste de sélection des dirigeants politiques depuis Périclès et les grandes décisions politiques sont toujours prises par des individus. Il n'en conclut pas pour autant à l'inutilité du parlement, comme l'écrit l'historien Wolfgang J. Mommsen ${ }^{(41)}$. Il est indispensable pour le vote du budget et des lois, et pour la publicité des mesures administratives. Il apporte la stabilité, garantit les droits des citoyens, contraint les aspirants au pouvoir à un travail parlementaire ordonné, contrôle le pouvoir et permet le renvoi pacifique du «dictateur plébiscitaire», s’il n'a plus la confiance des masses.

Max Weber répond ensuite aux critiques qui prétendent que la démocratisation favoriserait les méthodes démagogiques. Il souligne que la démagogie n’est pas caractéristique des États démocratiques, elle n'est pas absente des monarchies, même modérées: "Nous avons, en Allemagne, démagogie et influence de la populace sans démocratie, bien plus: du fait de l'absence de démocratie ordonnée " ${ }^{(42)}$. Il donne l'exemple de la propagande démagogique de l'amiral von Tirpitz pour la guerre sous-marine sans restriction. La "promotion démagogique» des chefs n'est pas liée au suffrage universel et se retrouve dans toutes les organisations, même dans l'armée comme le montre l'exemple de Moltke le Jeune. Mais la différence, c'est que l'homme politique peut être critiqué ouvertement par ses adversaires et par la presse pour ses méthodes démagogiques. Il ajoute que des partis fortement organisés, comme en Angleterre, sont un obstacle à la "démocratie de boulevard» qui menace, comme en France, lorsqu'il n'y a pas de "partis organisés rationnellement». Le processus de rationalisation des partis est en cours en Allemagne à gauche, mais aussi à droite avec l'apparition de la Vaterlandspartei. Il conclut le chapitre sur la nécessité de démocratiser le suffrage en Prusse pour mettre fin à des luttes stériles

40 Chap. V, «Parlamentarisierung und Demokratisierung», MWG, I, 15, p. 526-552.

41 Nous ne pouvons partager l'analyse de Wolfgang Mommsen, cf. Max Weber et la politique allemande 1890-1920 (note 14), p. 240-247. En fait, Max Weber associe un parlement fort et l'émergence de leaders "césaristes".

42 Ibid., p. 538. 
et rendre justice aux combattants: sinon «la nation ne se lèvera jamais plus comme en août 1914 contre une quelconque menace extérieure. Nous serions alors condamnés à rester un peuple continental [Binnenvolk] conservateur, peut-être bien administré d'un point de vue purement technique, mais sans possibilité - et, du reste aussi, sans aspiration intérieure à s'affirmer politiquement dans le monde» ${ }^{(43)}$.

\section{Les leçons de la crise de juillet 1917}

Le 7 septembre, Max Weber publie un nouvel article dans la Frankfurter Zeitung où il tire les «leçons de la crise de la chancellerie» de juillet 1917 qui a mené à la chute de Bethmann-Hollweg et à la résolution de paix du Reichstag du 19 juillet $^{(44)}$. Il estime que la crise est la conséquence de l'absence de système parlementaire et que «son déroulement fut un paradigme d'école»: «Le gouvernement allemand de fonctionnaires était face au Reichstag comme une force étrangère qui ne représentait que la bureaucratie publique [Beamtentum], mais ne tirait pas ses racines des partis politiques et ne pouvait ainsi les guider.» Il doute que le comité de parlementaires constitué par le chancelier Michaelis pour préparer la réponse à la note de paix du pape soit une solution, car la vraie question est: «Comment peut-on transformer en co-responsable politique un parlement condamné par sa structure interne actuelle à une politique négative? » Il critique vivement le lien, lors de la crise, entre la question de la paix et la question de la «nouvelle orientation" politique. Les réformes politiques n'ont rien à voir avec la question de la paix: «Ce que l'on appelle la "démocratisation" des institutions politiques allemandes, nous l'exigeons comme moyen indispensable pour maintenir l'unité de la nation au moment où nous allons peut-être vers une guerre défensive encore longue, la parlementarisation comme une garantie de l'unité de direction de la politique et pour éviter à l'avenir ces erreurs qui, dans le passé, n’ont pas été sans contribuer à la constitution de la coalition mondiale. » Il vitupère contre les "écrivassiers", responsables de la confusion entre paix et démocratisation: «Ce sont les discours inutiles et les gribouillis des écrivassiers qui les ont mises d'abord en relation. Depuis le début de la guerre, ils se sont efforcés de transformer notre combat national pour l'existence en un combat pour la structure étatique bureaucratique actuelle, soi-disant spécifiquement "allemande", contre une soi-disant "conspiration” des démocraties occidentales européennes. La grande majorité de la nation rejette [l'idée] que nos frères au dehors aient versé leur sang pour rien de mieux que ces produits d'écrivassiers et pour la domination de fonctionnaires sans contrôle qu'ils subliment.» Il rejette la thèse d'une conception proprement allemande de la démocratie et de la liberté, thème essentiel de la propagande des intellectuels allemands face à la propagande alliée sur la «libération de l'Allemagne de l'autocratie». Dans une lettre à Conrad Haußmann du même jour, il revient sur le lien malheureux entre paix et démocratisation: "Ce genre de propagande de "paix" est tout à fait inutile et nuit à la démocratisation. Il faut exiger et appliquer la démocratisation. Mais on lui nuit si on l'associe à la "paix". Si la paix ne vient pas malgré tout, alors la démocratie en sera blâmée.» Il craint, en fait, que la démocratie ne soit déconsidérée en cas de paix décevante. Il a le pressentiment de la future thèse du «coup de poignard dans le dos».

43 MWG, I, 15, p. 552.

44 «Die Lehren der deutschen Kanzlerkrisis», MWG, I, 15, p. 301-306. 
La question de la suppression du paragraphe 9,2 de la constitution est à nouveau abordée par la Commission principale du Reichstag le 25 août 1917, mais le chancelier Michaelis se prononce contre, tandis que le quotidien conservateur, la Kreuzzeitung, souligne qu'il y aurait «conflit de conscience» pour le chancelier qui pourrait recevoir comme plénipotentiaire prussien au Bundesrat des instructions contraires à ses convictions politiques de député. Dans un article anonyme de la Frankfurter Zeitung du 8 septembre, Max Weber répond à l'objection qu'un homme politique responsable démissionnerait en cas de conflit de conscience ${ }^{(45)}$. Il note, par ailleurs, que personne n'a soulevé d'objection lorsque le national libéral Eugen Schiffer a gardé son mandat au Landtag prussien après sa nomination comme sous-secrétaire d'État au Trésor. En revanche, il a dû démissionner de son mandat au Reichstag. Dans une lettre du 7 septembre à Conrad Haußmann où il annonce la publication de l'article, il insiste sur la notion de responsabilité politique: «Il ne peut qu'être souhaitable que par l'exercice d'un mandat au Reichstag soit renforcé le devoir de sacrifier la fonction à la conviction pour les fonctionnaires dirigeants du Reich. Ils doivent être des hommes politiques et non des "fonctionnaires" " ${ }^{(4)}$.

Max Weber revient sur la question du paragraphe 9,2 dans les Münchner Neueste Nachrichten des 15 et 17 octobre 1917 pour répondre aux réserves de la Bavière qui craint une remise en cause de l'équilibre fédéral ${ }^{(47)}$. Par une remarquable analyse de la constitution, il démontre que la Bavière ne peut s'opposer à une parlementarisation du Reich et à une évolution de son fonctionnement du fait de l'introduction du suffrage universel égal en Prusse. L'empereur a en effet toute liberté pour choisir un chancelier et des secrétaires d'État dans la majorité du Reichstag sans consulter le Bundesrat. Avec le paragraphe 9,2 qui interdit au chancelier d'être membre du Reichs$\operatorname{tag}^{(48)}$, le pouvoir du chancelier repose en fait sur le Landtag prussien devant lequel le ministère prussien, dont il est membre comme ministre des Affaires étrangères, est responsable. Avec le suffrage universel égal, l'influence du Landtag sur le ministère, et donc sur le chancelier, sera encore renforcée. Ainsi, tant que le paragraphe 9,2 sera en vigueur, le chancelier dépendra de la Prusse et présidera le Bundesrat en tant que représentant de la Prusse. Cela renforcera la centralisation au profit de la Prusse, et la Bavière perdra toute influence effective sur la politique du Reich. Max Weber conclut que les États allemands ne pourront avoir d'influence politique que dans le cadre d'une parlementarisation, c'est-à-dire par leurs députés au Reichstag, mais à condition que le chancelier puisse en être membre et en dépendre directement. Il envisage aussi une forme de responsabilité du gouvernement du Reich à l'égard du Bundesrat par le biais de commissions du Bundesrat qui seraient consultées avant toute décision importante. L'alternative est donc simple: le maintien du paragraphe 9,2 et un chancelier dépendant de la Prusse, ce qui signifierait l'évolution vers une grande Prusse, ou la parlementarisation qui préserverait l'influence des États allemands par le biais de leurs élus au Reichstag.

45 «Die Abänderung des Artikels 9 der Reichsverfassung», MWG, I, 15, p. 310-313.

46 MWG, II, 9, lettre du 7 septembre en réponse à la lettre de Haußmann du 3 septembre.

47 «Bayern und die Parlementarisierung im Reich», MWG, I, 15, p. 330-338.

48 L'article 15 de la constitution précise que le chancelier préside le Bundesrat. 


\section{Max Weber et «la forme future de l'État allemand»}

Max Weber publie, fin novembre et début décembre 1918, une série de cinq articles sur la forme constitutionnelle future de l'État allemand dans la Frankfurter Zeitung. Ces articles sont publiés, très légèrement remaniés, sous forme de brochure le 14 janvier 1919 dans le cadre de la campagne pour l'élection à l'Assemblée nationale constituante du parti démocratique allemand qu'il a contribué à fonder ${ }^{(49)}$.

Dans la préface de la brochure, Max Weber précise que ses propositions de réforme antérieures sont dépassées par la situation. Il s'agissait alors de parlementariser dans le cadre de la monarchie constitutionnelle sous hégémonie prussienne. Il considère toujours la monarchie parlementaire comme le meilleur régime, mais la dynastie prussienne n'a su empêcher une dictature militaire et s'est définitivement déconsidérée par les interventions intempestives du monarque. Le parlement a été à ce point rabaissé qu'il n’a pas de racines profondes et a été ainsi dépassé par une minorité de révolutionnaires. Aujourd'hui, il s'agit de reconstruire de manière rationnelle par une constituante. La république s'impose pour éviter une guerre civile et l'intervention de l'étranger. Elle permettra de réunir le plus d'Allemands, y compris les Autrichiens. Elle contraindra la bourgeoisie à prendre ses responsabilités, car on ne pourra instaurer d'ordre durable et préserver les conquêtes démocratiques sans sa libre collaboration. Alors qu'il laisse entendre qu'il est plutôt favorable à une solution unitaire, il se prononce pour un État fédéral, car les États allemands, en particulier la Bavière et l'Autriche, n'accepteraient pas une centralisation par la Prusse, mais aussi parce que les Alliés s'y opposeraient. L'objectif de son esquisse est de montrer qu'une constitution fédérale ne reposant pas sur une base dynastique est possible. Il a aussi à l'esprit les projets de socialisation, la pénétration de l'État dans l'économie et la fusion de la bureaucratie publique avec celle de grandes entreprises, qui menacent de réduire la capacité du pouvoir politique à contenir le pouvoir économique, comme l'a montré l'économie de guerre ${ }^{(50)}$.

Max Weber présente ensuite les solutions institutionnelles possibles dans le cadre d'une république fédérale grande-allemande, où il n'y aurait plus de lien privilégié entre la Prusse et le Reich. Il pose deux questions fondamentales: la structure du gouvernement du Reich, la représentation des États fédérés. Après avoir envisagé une assemblée fédérale élue par le peuple, comme aux États-Unis, ou par les parlements des États, il estime que le système des délégués instruits par les gouvernements des États, comme dans l'ancien Bundesrat, est le plus simple, à condition de supprimer les petits États, de rééquilibrer les voix entre États, de renforcer leurs droits particuliers inaliénables et d'introduire une alternance à la présidence de l'assemblée. Il envisage trois possibilités pour le gouvernement du Reich: «Une tête du Reich plébiscitaire,

49 Deutschlands künftige Staatsform, Flugschriften der Frankfurter Zeitung, 1919, 40 p. Les articles sont publiés le 22, 24, 28 et 30 novembre, ainsi que le 5 décembre, alors qu'on ne connaît pas encore la date des élections à l'assemblée constituante, fixée au 19 janvier par le congrès des soviets le 19 décembre, cf. Christian BAechler, L'Allemagne de Weimar 1919-1933, Paris, 2007, p. 72-75. Sur l'activité politique de Weber, cf. W.J. Mommsen, Max Weber et la politique allemande 1890-1920 (note 14), p. 388-396.

50 Dans une conférence à Vienne sur le socialisme, en juin 1918, où il analyse la révolution bolchevique devant un auditoire d'officiers autrichiens, Max Weber souligne cette menace. 
parlementaire ou fédérale?» Il ne cache pas sa préférence pour la première solution, surtout dans le cas d'une représentation des États par des délégués - la solution d'un Bundesrat -, car il estime qu'il ne pourrait y avoir alors de vrai parlementarisme au niveau de la fédération: «Un président du Reich s'appuyant sur la légitimité révolutionnaire du vote populaire, faisant face aux corps du Reich [Reichstag et Bundesrat] avec ses droits propres, aurait une tout autre autorité que celle d'un élu par voie parlementaire.» Un mandat long serait gage de continuité, tout particulièrement nécessaire dans l'hypothèse de socialisations. Les divergences entre le président et le gouvernement responsable devant le Reichstag se règleraient au cas par cas, selon l'équilibre des forces du moment.

Il rejette le suffrage proportionnel pour l'élection du Reichstag, car il n'y aurait plus de direction politique unie, mais des compromis entre partis. Il se prononce aussi contre les référendums, car il estime qu'ils ont une fonction conservatrice et renforcent ainsi le pouvoir de la bureaucratie. Associer référendum, suffrage proportionnel et présidence plébiscitaire, c'est réduire le rôle du parlement au profit de la bureaucratie: «Le parlement ne serait plus alors le lieu de sélection des chefs.» Contrairement à l'interprétation de Wolfgang J. Mommsen, Max Weber ne verse pas dans la promotion d'un pouvoir charismatique exclusif et ne réduit pas le rôle du parlement à une politique strictement négative, comme sous l'Ancien Régime ${ }^{(51)}$. Le parlement est le lieu de sélection des chefs, il contrôle les ministres et constitue un garde-fou contre le pouvoir personnel du président grâce au droit d'initiative d'un référendum de révocation. Il est aussi, avec le président élu, un contrepoids à la bureaucratie, comme dans les écrits antérieurs de Max Weber.

\section{Conclusion}

Dès le début de la guerre, Max Weber a voulu contribuer à l'effort de guerre en s'engageant. Trop âgé pour être placé dans l'armée active, il doit se contenter d'une fonction dans la commission gérant les hôpitaux de la région de Heidelberg jusqu'à septembre 1915. Il aspire ensuite à un rôle actif dans la définition de la politique allemande, mais doit se contenter d'une activité de publiciste. Il intervient dans la question des buts de guerre et dans le débat sur la guerre sous-marine. Pessimiste sur l'issue de la guerre, en particulier après l'entrée en guerre des États-Unis, et persuadé qu'on ne pourra poursuivre la lutte et préserver l'avenir de l'Allemagne qu'en réformant le régime politique, il mène à partir de 1917 une campagne souvent très violente en faveur des réformes en Prusse et au niveau du Reich. Malgré la censure, plus attentive à ses critiques à la suite de l'article dans la Frankfurter Zeitung du 24 juin, il poursuit sa campagne, à l'occasion sous l'anonymat. Son activité de publiciste a peu d'effets immédiats, mais ses écrits sont une contribution essentielle à la réflexion sur l'organisation de l’État allemand après la révolution de novembre 1918.

51 W.J. Mommsen, Max Weber et la politique allemande 1890-1920 (note 14), p. 421-437, 477-486. Dans un article publié en 2001, Mommsen donne une interprétation plus nuancée du programme de Weber, cf. "Politik im Vorfeld der "Hörigkeit der Zukunft". Politische Aspekte der Herrschaftssoziologie Max Webers", in: Edith Hanke, Wolfgang J. Mommsen (éd.), Max Webers Herrschaftssoziologie, Tübingen, 2001, p. 303-319. 
On constate dans les écrits de 1917 et de 1918 un mélange de réactions passionnelles à l'actualité récente et immédiate, qui trahissent le tempérament volcanique de l'ardent patriote qu'est Max Weber, et de considérations générales sur l'évolution de la société moderne, nourries par ses travaux scientifiques. La question de la rationalisation et de la bureaucratisation croissante de la société moderne est présente dans tous ses écrits avec comme interrogation sous-jacente la préservation d'un espace de liberté, de responsabilité. La coexistence d'un secteur étatique et d'une économie de marché est, pour lui, un facteur essentiel de préservation de la liberté au niveau de la société globale. L'erreur du socialisme est de supprimer le contrepoids de l'entreprise privée. L'expérience de l'économie de guerre avec l'intervention de la bureaucratie publique ne peut que le confirmer dans la nécessité du marché comme facteur de rationalisation et d'allocation des ressources.

Max Weber s'interroge pendant la guerre sur les solutions institutionnelles permettant de concilier dans l'État moderne la nécessité d'une bureaucratie efficace avec la légitimité de la démocratie de masse, qui lui apparaît comme un phénomène inéluctable ${ }^{(52)}$. Dans le cadre de la monarchie constitutionnelle, le parlement doit jouer le rôle de contrepoids à la bureaucratie publique grâce au droit d'enquête, instrument de contrôle de la bureaucratie et du gouvernement, mais aussi lieu de formation des hommes politiques. La compétition entre les partis, qui eux-mêmes se bureaucratisent pour mener une propagande de masse, est un facteur de préservation des libertés et permet la sélection de leaders politiques responsables. La lutte est un élément fondamental de la politique, de même que la concurrence pour l'économie. Dès 1917, il insiste sur l'aspect "plébiscitaire», "césariste» de la sélection des leaders dans la démocratie de masse. La dimension "plébiscitaire» de la politique est le résultat de la démocratisation. Les masses ne sont plus simples objets passifs, mais veulent peser sur les décisions, d'où une nouvelle forme de légitimité du pouvoir. Après la défaite et la révolution, il prend en compte le fait que les dynastes allemands sont totalement déconsidérés. Aussi cherche-t-il dans le cadre républicain, dans le contexte de la reconversion économique et d'une socialisation partielle, un contrepoids supplémentaire à une bureaucratisation renforcée. C’est le rôle du président de la République élu au suffrage universel, dont la légitimité est charismatique. La tendance plébiscitaire de la démocratie de masse s'oppose ainsi à la bureaucratisation de la société. Il est probable que l'exemple de chefs de guerre tels que Lloyd George et Clemenceau, ainsi que la fonction charismatique de Hindenburg depuis 1915, contribuent à une réévaluation du rôle du charisme dont Max Weber croyait le déclin inéluctable dans la société moderne ${ }^{(53)}$.

52 Dans sa nécrologie de la Frankfurter Zeitung du 20 juin 1920, Ernst Troeltsch insiste sur le fait que Max Weber est un démocrate de raison: «La démocratie n'était pour lui que la destinée du monde moderne et signifiait la perte de grandeurs et beautés infinies.» Troeltsch souligne aussi l'ardent patriotisme qui explique la violence de ton des écrits de guerre.

53 Dans la Herrschaftssoziologie rédigée avant la guerre. Cf. F. CHAzEL, «Les Écrits politiques de Max Weber» (note 12), p. 867-870, ainsi que Stefan Breuer, «Das Charisma des Führers», in: ID. (éd.), Bürokratie und Charisma. Zur Politischen Soziologie Max Webers, Darmstadt, 1994, p. 144-175. Pour Stefan Breuer, Max Weber tente de «domestiquer les tendances charismatiques de la démocratie de masse». 
À l'arrière-plan de ces réflexions générales sur la démocratisation, il y a dans tous les écrits de guerre de Max Weber le souci de mobiliser les masses dans l'effort de guerre, puis pour la reconstruction de l'Allemagne qu'il prévoit difficile. Le président de la République, «homme de confiance des masses», pourra imposer l'unité de direction indispensable pour la reconstruction de la "puissance» (Macht) de l'Allemagne. La grandeur nationale, plus que la démocratie, est la valeur suprême pour lui, dans la continuité de sa leçon d'introduction à l'Université de Fribourg de mai 1895 sur «l'État national et la politique économique» ${ }^{(54)}$.

\title{
Résumé
}

Pessimiste sur l'issue de la guerre, Max Weber est persuadé qu'on ne peut mobiliser la population dans l'effort de guerre, puis dans la reconstruction de l'Allemagne qu'il prévoit difficile, qu'en réformant immédiatement le régime politique. Aussi mène-t-il à partir de mars 1917, et jusqu'à la fin de la guerre, une campagne de presse souvent violente en faveur de la démocratisation du mode de scrutin prussien et de la parlementarisation $d u$ Reich. Il s'interroge aussi, dans la continuité de ses réflexions d'avant-guerre sur la bureaucratisation croissante de la société moderne, sur les solutions institutionnelles permettant de concilier la nécessité d'une bureaucratie efficace avec la légitimité de la démocratie de masse. Il se prononce pour une monarchie parlementaire avec un parlement renforcé, contrepoids à la bureaucratie publique et lieu de formation et de sélection des chefs politiques, même s'il insiste dès 1917 sur l'aspect "plébiscitaire" inévitable de la sélection des chefs dans la démocratie de masse. Après la chute de la monarchie, il se prononce pour un président de la République élu au suffrage universel, dont la légitimité charismatique ferait contrepoids à la bureaucratie, mais avec un parlement fort comme garde-fou contre un pouvoir personnel. Il rejette son élection au suffrage proportionnel qui le rendrait inefficace, et le référendum qui renforcerait le conservatisme et le pouvoir de la bureaucratie.

\begin{abstract}
As he is pessimistic about the outcome of the war, Max Weber is convinced that the population can't be mobilized in the war effort and then in the reconstruction of Germany - which he predicts to be difficult - unless the political regime is reformed immediately. That's why, from March 1917 to the end of the war, he leads a rather harsh press campaign in favour of the democratization of the voting system of Prussia and the change towards a parliamentary system of the Reich. Developing his pre-war ideas about the growing bureaucratization of modern society, he also wonders about the institutional solutions which would allow reconciling the necessity of an efficient bureaucracy with the legitimacy of mass democracy. He favours a parliamentary monarchy with a reinforced Parliament acting as a counterbalance to public bureaucracy and being a place for training and selecting political leaders; yet, as early as 1917, he insists on the inevitable "plebiscitary" aspect of the selection of the leaders in mass democracy. After
\end{abstract}

54 Nous renvoyons aux analyses de Raymond Aron sur Max Weber et son œuvre dans Les étapes de la pensée sociologique, Paris, 1967, en particulier sa conclusion, p. 564-571. 
the fall of the monarchy, he favours a President of the Republic elected by universal suffrage - the charismatic legitimacy of which would counterbalance bureaucracy along with a strong parliament as a safeguard against personal power. He rejects both the idea of an election by proportional representation which would make the parliament inefficient, and the idea of a referendum which would reinforce conservatism and the power of bureaucracy. 